TRANSACTIONS OF THE

AMERICAN MATHEMATICAL SOCIETY

Volume 171, September 1972

\title{
RADIAL LIMIT SETS ON THE TORUS
}

BY

\section{LAURENCE D. HOFFMANN( ${ }^{1}$ )}

\begin{abstract}
Let $U^{N}$ denote the unit polydisc and $T^{N}$ the unit torus in the space of $N$ complex variables. A subset $A$ of $T^{N}$ is called an (RL)-set (radial limit set) if to each positive continuous function $\rho$ on $T^{N}$, there corresponds a function $f$ in $H^{\infty}\left(U^{N}\right)$ such that the radial limit $|f|^{*}$ of the absolute value of $f$ equals $\rho$, a.e. on $T^{N}$ and everywhere on $A$. If $N>1$, the question of characterizing ( $R L$ )-sets is ope $n$, but two positive results are obtained. In particular, it is shown that $T^{N}$ contains an (RL)-set which is homeomorphic to a cartesian product $K \times T^{N-1}$, where $K$ is a Cantor set. Also, certain countable unions of "parallel" copies of $T^{N-1}$ are shown to be (RL)-sets in $T^{N}$. In one variable, every subset of $T$ is an (RL)-set; in fact, there is always a zero-free function $f$ in $H^{\infty}(U)$ with the required properties. It is shown, however, that there exist a circle $A \subset T^{2}$ and a positive continuous function $\rho$ on $T^{2}$ to which correspond no zero-free $f$ in $H^{\infty}\left(U^{2}\right)$ with $|f|^{*}=\rho$ a.e. on $T^{2}$ and everywhere on $A$.
\end{abstract}

1. Introduction. To each bounded, nonnegative function $\rho$ on the unit circle $T$ with $\log \rho \in L^{1}(T)$, there corresponds a bounded holomorphic function $f$ on the unit disc $U$ for which the radial limit $|f|^{*}$ of the absolute value of $f$ equals $\rho$ a.e. on $T$ [3, p. 54]. It is known that this result does not generalize to the unit polydisc $U^{N}$ in the space of $N$ complex variables. However, one positive result due to Rudin [3, p. 55] asserts that if $\rho$ is positive, bounded and lower semicontinuous on the unit torus $T^{N}$, there exists a function $f$ in $H^{\infty}\left(U^{N}\right)$ with $|f|^{*^{*}}=\rho$ a.e. on $T^{N}$. In this paper, a modification of Rudin's construction will be used to obtain more precise information about the sets on which the equality $|f|^{*}=\rho$ is satisfied. In particular, the following related class of sets will be considered.

Definition. A subset $A$ of $T^{N}$ is called an ( $\left.R L\right)$-set (radial limit set) if to each positive continuous function $\rho$ on $T^{N}$, there corresponds a function $f$ in $H^{\infty}\left(U^{N}\right)$ with $|f|^{*}=\rho$ a.e. on $T^{N}$ and everywhere on $A$.

In one variable, every subset of $T$ is an (RL)-set. Indeed, if $f$ is the outer function 1971.

Presented to the Society, November 20,1971; received by the editors December 1 ,

AMS 1970 subject classifications. Primary 32A10, 30A88, 30 A 78.

Key words and phrases. Polydisc, torus, Hardy space $H^{\infty}\left(U^{N}\right)$, radial limits, radial limit sets, outer functions, inner functions.

(1) Parts of this paper appear in the author's dissertation written under the supervision of Professor Walter Rudin at the University of Wisconsin. 


$$
f(z)=\exp \left\{\int_{T} \frac{w+z}{w-z} \log \rho(w) d m(w)\right\}
$$

the continuity of $\rho$ implies that $|f|^{*}(w)=\rho(w)$ for all $w \in T$. In several variables the question of characterizing (RL)-sets is open. However, in $\$ 2$ of this paper, two types of (RL)-sets will be identified. In $\$ 3$, some differences between one and several variables are discussed regarding the possibility of choosing a zero-free function $f$ in the definition of (RL)-sets.

2. Construction of radial limit sets. The purpose of this section is to prove the following theorems which identify two types of (RL)-sets.

The ore m 1. Let $a=\left(a_{1}, \ldots, a_{N}\right)$ be a point in $Z^{N}$ with $a_{j}>0$ for $1 \leq j \leq$ $N$, and $\left\{p_{k}\right\}$ a sequence of complex numbers with $\left|p_{k}\right|=1$.

If $A_{k}=\left\{w \in T^{N}: w^{a}=p_{k}\right\}$, for $k=1,2, \cdots$, then $A=\bigcup A_{k}$ is an $(R L)$-set in $T^{N}$.

(As usual, $Z^{N}$ denotes the space of lattice points $a=\left(a_{1}, \ldots, a_{N}\right)$ where each $a_{j}$ is an integer. If $w=\left(w_{1}, \ldots, w_{N}\right)$ is in $T^{N}, w^{a}$ stands for the mono$\operatorname{mial} w_{1}^{a_{1}} \ldots w_{N}^{a}{ }^{\prime}$ )

The ore $\mathrm{m}$ 2. Suppose $K$ is the usual "middle-tbird" Cantor set on $[0,1]$, and $S=\phi(K)$ where

$$
\phi(t)=-\exp (2 \pi i t), \quad(0 \leq t \leq 1)
$$

If $A=\left\{w \in T^{N}: w_{1} w_{2} \cdots w_{N} \in S\right\}$, then $A$ is an (RL)-set in $T^{N}$.

In Theorem 1, each set $A_{k}$ consists of a finite number of "parallel"' $(N-1)$ dimensional tori. Hence, the theorem asserts that any countable union of copies of $T^{N-1}$ which are parallel to $\left\{w \in T^{N}: w^{a}=1\right\}$ is an (RL)-set in $T^{N}$. Theorem 2 says that $T^{N}$ contains an (RL)-set which is topologically the cartesian product of a Cantor set and an $(N-1)$-dimensional torus.

The first lemma is essentially Rudin's "modification theorem" [3, The orem 2.4.2] upon which the construction of (RL)-sets will be based. Since the conclusion of the lemma is somewhat more detailed than Rudin's original version, a proof will be sketched.

As in [3, Chapter 2], $R P\left(T^{N}\right)$ will be the class of all complex Borel measures $\mu$ on $T^{N}$ whose Poisson integral $P[d \mu]$ is the real part of a holomorphic function in $U^{N}$. RP-measures are characterized by the vanishing of their Fourier coefficients outside the positive and negative cones of $Z^{N}$.

If $Q$ is a trigonometric polynomial on $T^{N}, \operatorname{deg}(Q)$ will denote the smallest positive integer $d$ such that the Fourier coefficient $\hat{Q}(a)$ vanishes whenever $a=$ $\left(a_{1}, \cdots, a_{N}\right) \in Z^{N}$ with $\left|a_{j}\right|>d$ for some $j$. 
Lemma 1. Suppose $\beta \in T^{N}$ and $s \in Z^{N}$ with $s_{j}>0$ for $1 \leq j \leq N$. Let $E=$ $\left\{w \in T^{N}: w^{s}=1\right\}$, and $F=\beta E=\left\{\left(\beta_{1} w_{1}, \cdots, \beta_{N} w_{N}\right): w \in E\right\}$. Let $\nu$ denote the Haar measure for the compact topological group $E$, and let $\mu$ be the translation of $\nu$ to the coset $F$; i.e., $\mu(A)=\nu(\bar{\beta} A)$. If $Q$ is a nonnegative trigonometric polynomial on $T^{N}$ with $\operatorname{deg}(Q)<s_{j}$ for $1 \leq j \leq N$, then

(a) $Q-Q d \mu \in R P\left(T^{N}\right)$,

(b) $\hat{Q}(0)=(Q d \mu)^{\wedge}(0)$, and

(c) $\|Q d \mu\|=\|Q\|_{1}$.

Proof. For $a \in Z^{N}$, the Fourier coefficients of $\mu$ and $\nu$ are related by

$$
\hat{\mu}(a)=\bar{\beta}^{a} \hat{\nu}(a) \text {. }
$$

The function $\bar{w}^{a}$ is a character on $E$ and is identically 1 on $E$ if and only if $a=$ $k s$ for some integer $k$. Since $\nu$ is the Haar measure for $E$, it follows from (2) that

$$
\hat{\mu}(a)= \begin{cases}\bar{\beta}^{a} & \text { if } a=k s \text { for some } k \in Z, \\ 0 & \text { otherwise }\end{cases}
$$

Let $Y_{N}=Z_{+}^{N} \cup\left(-Z_{+}^{N}\right)$ where $Z_{+}^{N}$ is the positive cone of all $a \in Z^{N}$ with $a_{j} \geq 0$ for $1 \leq j \leq N$. If $Q$ is a nonnegative trigonometric polynomial on $T^{N}, \hat{Q}(a)$ $=0$ except for $a$ in some finite set $X \subset Z^{N}$. Thus

$$
0 \notin X+k s \subset Y_{N}, \quad \text { for } k= \pm 1, \pm 2, \ldots
$$

whenever $s_{j}>\operatorname{deg}(Q)$ for $1 \leq j \leq N$.

It follows from (3) that

$$
(Q d \mu)^{\hat{\imath}}(a)=\sum_{n \in X} \hat{Q}(n) \hat{\mu}(a-n)=\sum_{k=-\infty}^{\infty} \hat{Q}(a-k s) \bar{\beta}^{k s} .
$$

If $a \notin Y_{N}$ and $k \neq 0,(4)$ implies $a-k s \notin X$ so that $\hat{Q}(a-k s)=0$. Hence, by (5), $(Q d \mu) \wedge(a)=\hat{Q}(a)$ for all $a \notin Y_{N}$, which says that $Q-Q d \mu$ is in $R P\left(T^{N}\right)$.

Finally, it follows from (4) and (5) that

$$
(Q d \mu)^{\wedge}(0)=\hat{Q}(0)
$$

while $Q \geq 0$ implies $\|Q d \mu\|=(Q d \mu)^{\wedge}(0)$ and $\hat{Q}(0)=\|Q\|_{1}$. Hence, by (6), $\|Q d \mu\|$ $=\|Q\|_{1}$, and the proof is complete.

Le mma 2. Let $\beta, s$, and $\mu$ be defined as in Lemma 1. If $r=\left(r_{1}, \cdots, r_{N}\right)$ with $0<r_{j}<1$, and $w=\left(w_{1}, \cdots, w_{N}\right) \in T^{N}$, then the Poisson integral of $\mu$ is given by

$$
P[d \mu](r w)=\mathscr{P}\left((r w \bar{\beta})^{s}\right),
$$

where $\mathcal{P}$ is the Poisson kernel in one variable,

$$
\mathcal{P}(\zeta)=\operatorname{Re}[(1+\zeta) /(1-\zeta)] \quad(\zeta \in U)
$$


and

$$
(r w \bar{\beta})^{s}=\prod_{n=1}^{N}\left(r_{n} w_{n} \bar{\beta}_{n}\right)^{s}
$$

Proof. The familiar series expansion [3, p. 17] for the Poisson kernel is combined with (3) to give

$$
\begin{aligned}
P[d \mu](r w) & =\sum_{k=-\infty}^{\infty} \bar{\beta}^{k s} r_{1}^{|k| s_{1}} \ldots r_{N}^{|k| s_{N}} w^{k s} \\
& \left.=\operatorname{Re}\left\{\left[1+(r w \bar{\beta})^{s}\right] /[1-r w \bar{\beta})^{s}\right]\right\} .
\end{aligned}
$$

The next lemma follows immediately from Lemma 2 and well-known properties of the Poisson kernel in one variable [2, p. 224].

Lemma 3. Suppose $\beta, s, \mu, r$ and $w$ are defined as before, and let

$$
\Gamma(\delta)=\left\{e^{i \theta}: 2 \pi \delta \leq \theta \leq 2 \pi(1-\delta)\right\},
$$

and

$$
M(\delta)=\sup \left\{\mathcal{P}\left(\operatorname{Re}^{i \theta}\right): 0<R<1 \text { and } e^{i \theta} \in \Gamma(\delta)\right\}
$$

Then,

(a) $P[d \mu](r w) \leq M(\delta)<\infty$ if $0<\delta<1 / 2$ and $(\omega \bar{\beta})^{s} \in \Gamma(\delta)$, and

(b) $\lim _{r \rightarrow 1} P\left[\overline{d \mu} \mu(r w)=0\right.$ whenever $(w \bar{\beta})^{s} \neq 1$.

Lemma 4. Suppose $p_{1}, \cdots, p_{n}$ and $q_{1}, q_{2}, \cdots$ are points of $T$, and let $\Gamma$ be a nondegenerate arc on $T$. To each number $\eta>0$, there corresponds an integer $d>\eta$ and a point $\gamma \in T$ for whicb

(a) $\left(p_{k} \gamma\right)^{d} \in \Gamma$ for $1 \leq k \leq n$, and

(b) $1 \notin\left\{\left(q_{j} \gamma\right)^{d}: 1 \leq j<\infty\right\}$.

Proof. A classical theorem of Dirichlet [4, Volume I, p. 235] implies that for $\epsilon>0$, there exists an integer $d>\eta$ for which $\left|1-\left(p_{k}\right)^{d}\right|<\epsilon$ for $1 \leq k \leq n$. It follows that if $\epsilon$ is sufficiently small, there is an open arc $\Lambda$ on $T$ such that $\left(p_{k}\right)^{d} \lambda \in \Gamma$ whenever $\lambda \in \Lambda$. Since there are uncountably many points in $T$ with $\gamma^{d} \in \Lambda$, such a point can be chosen so that none of the points $\left(q_{j} \gamma\right)^{d}$ (for $1 \leq$ $j<\infty)$ is equal to 1 .

The proof of Rudin's boundary value theorem [3, Theorem 3.5.3] can now be modified to establish Theorem 1.

Proof of The orem 1. Let $\rho$ be a positive continuous function on $T^{N}$ and assume without loss of generality that $\log \rho>0$. Choose nonnegative trigonometric polynomials $Q_{n}$ on $T^{N}$ such that $\log \rho=\Sigma_{n=1}^{\infty} Q_{n}$ on $T^{N}$, and, for $n=1,2, \ldots$,

$$
\left\|Q_{n}\right\|_{\infty} \leq 2^{1-n}\|\log \rho\|_{\infty} \text {. }
$$

Fix $\delta$ with $0<\delta<1 / 2$. For each $n=1,2, \ldots$, Lemma 4 implies that there exist an integer $d_{n}>\operatorname{deg}\left(Q_{n}\right)$ and a point $\gamma_{n} \in T$ such that 


$$
\left(p_{k} \gamma_{n}\right)^{d} n \in \Gamma(\delta) \quad \text { for } 1 \leq k \leq n
$$

and

$$
1 \not\left\{\left(p_{j} \gamma_{n}\right)^{d}{ }_{n}: 1 \leq j<\infty\right\}
$$

Let $s_{n}=d_{n} a \in Z^{N}$ and choose $\beta_{n} \in T^{N}$ with $\left(\bar{\beta}_{n}\right)^{a}=\gamma_{n}$. As in Lemma 1 , let $E_{n}=\left\{w \in T^{N}: w^{s} n=1\right\}, F_{n}=\beta_{n} E_{n}, \nu_{n}$ the Haar measure for $E_{n}$, and $\mu_{n}$ the translation of $\nu_{n}$ to the coset $F_{n}$. Since $d_{n}>\operatorname{deg}\left(Q_{n}\right)$, it follows from Lemma 1 that

and

$$
Q_{n}-Q_{n} d \mu_{n} \in R P\left(T^{N}\right) \text { for } n=1,2, \cdots,
$$

$$
\left\|Q_{n} d \mu_{n}\right\|=\left\|Q_{n}\right\|_{1} \quad \text { for } n=1,2, \ldots .
$$

Let $d \sigma_{n}=Q_{n} d \mu_{n}$. The trigonometric polynomials $Q_{n}$ are nonnegative so that

$$
\sum\left\|\sigma_{n}\right\|=\sum\left\|Q_{n}\right\|_{1}=\int \sum Q_{n}=\int \log \rho<\infty,
$$

and the series $\Sigma \sigma_{n}$ converges in total variation norm to a positive measure $\sigma$. Since each $\sigma_{n}$ is singular (with respect to the Haar measure of $T^{N}$ ), so is $\sigma$. Moreover, if a lies outside the union of the positive and negative cones of $Z^{N}$, then

$$
\hat{\sigma}(\alpha)=\sum \hat{\sigma}_{n}(\alpha)=\sum \hat{Q}_{n}(\alpha)=(\log \rho) \hat{(\alpha)},
$$

so that $\log \rho-d \sigma$ is in $R P\left(T^{N}\right)$. In particular, there exists a holomorphic function $g$ on $U^{N}$ with

$$
\operatorname{Re}[g]=P[\log \rho-d \sigma] .
$$

Define $f=e^{g}$. Clearly, $f$ is in $H^{\infty}\left(U^{N}\right)$ since $\log \rho$ is bounded above and $\sigma>0$. Also, $|f|^{*}=\rho$ a. e. on $T^{N}$. In fact, the continuity of $\rho$ implies

$$
\lim _{r \rightarrow 1} P[\log \rho](r w)=\log \rho(w), \quad \text { for all } w \in T^{N},
$$

hence $|f|^{*}(w)=\rho(w)$ if and only if

$$
\lim _{r \rightarrow 1} P[d \sigma](r w)=0 .
$$

Thus, it remains to show that $s_{n}$ and $\beta_{n}$ have been chosen so that (10) holds for all $w \in A$.

If $w \in A_{k}$, then $w^{a}=p_{k}$ and it follows from the choice of $s_{n}$ and $\beta_{n}$ that

$$
\left(w \bar{\beta}_{n}\right)^{s} n=\left(p_{k} Y_{n}\right)^{d n}
$$

for $n=1,2, \ldots$. Hence (8) implies 


$$
\left(w \bar{\beta}_{n}\right)^{s} n \in \Gamma(\delta) \quad \text { for } w \in A_{k} \text { and } n \geq k
$$

while (9) gives

$$
\left(w \bar{\beta}_{n}\right)^{s} n \neq 1 \text { for } w \in A \text { and } n=1,2, \ldots
$$

Since

$$
P\left[d \sigma_{n}\right](r w) \leq\left\|Q_{n}\right\|_{\infty} P\left[d \mu_{n}\right](r w),
$$

it now follows from (7), (12), and Lemma 3 that

$$
P\left[d \sigma_{n}\right](r w) \leq 2^{1-n} M(\delta)\|\log \rho\|_{\infty}
$$

for all $w \in A_{k}$ and $n \geq k$. Hence, for each $w \in A_{k}$, the series $\sum_{n=1}^{\infty} P\left[d \sigma_{n}\right](r w)$ converges uniformly in $r$ for $0<r<1$, and so

$$
\begin{aligned}
\lim _{r \rightarrow 1} P[d \sigma](r w) & =\lim _{r \rightarrow 1} P\left[d \sigma_{n}\right](r w)=\sum \lim _{r \rightarrow 1} P\left[d \sigma_{n}\right](r w) \\
& \leq \sum 2^{1-n}\|\log \rho\|_{\infty} \lim _{r \rightarrow 1} P\left[d \mu_{n}\right](r w) .
\end{aligned}
$$

Finally, (13) and Lemma 3 imply that for each $k=1,2, \ldots, \lim _{r \rightarrow 1} P\left[d \mu_{n}\right](r w)$ $=0$ if $w \in A_{k}$ and $n=1,2, \ldots$, so that by $(15), \lim _{r \rightarrow 1} P[d \sigma](r w)=0$ for all $w \in A$, and the proof is complete.

Proof of Theorem 2. Let $\rho$ be continuous on $T^{N}$ with $\log \rho>0$, and choose nonnegative trigonometric polynomials $Q_{n}$ such that $\log \rho=\Sigma Q_{n}$, and $\left\|Q_{n}\right\|_{\infty} \leq$ $2^{1-n}\|\log \rho\|_{\infty}$. For each $n=1,2, \ldots$, choose an integer $k_{n}$ such that $3^{k_{n}}>$ $\operatorname{deg}\left(Q_{n}\right)$ and let $d_{n}=3^{k_{n}}$. Let $E_{n}=\left\{w \in T^{N}:\left(w_{1} w_{2} \cdots w_{N}\right)^{d_{n}}=1\right\}, \nu_{n}=$ Haar measure for $E_{n}$, and $d \sigma_{n}=Q_{n} d \nu_{n}$. If $\sigma$ and $f$ are now defined as in the proof of Theorem 1 , it remains to show only that $\lim _{r \rightarrow 1} P[d \sigma](r w)=0$ for all $w \epsilon$ $A$. This will follow exactly as in Theorem 1 from the following estimate:

$$
P\left[d \nu_{n}\right](r w) \leq M(1 / 6) \text { for } w \in A, 0<r<1 \text {, and } n=1,2, \cdots,
$$

where $M(1 / 6)$ is the supremum defined in Lemma 3.

To verify (16), observe that $\lambda \in S$ if and only if

$$
\lambda^{3^{k}} \in \Gamma(1 / 6) \quad \text { for each } k=0,1,2, \ldots .
$$

If $w$ is in $A$, then $w_{1} w_{2} \cdots w_{N}$ is in $S$; in particular, by (17), $\left(w_{1} w_{2} \cdots w_{N}\right)^{d} n$ $\epsilon \Gamma(1 / 6)$ for $n=1,2, \ldots$, and (16) follows from Lemma 3.

3. Zero-free functions. In one variable, the unit circle is an (RL)-set. In fact, the function (1) corresponding to the positive continuous function $\rho$ on $T$ has the additional property that it never vanishes in $U$. Whether the torus $T^{N}$ is also an (RL)-set when $N>1$ is an open question. However, the next theorem shows that in general the possibility of choosing a zero-free function in the definition 
of (RL)-sets does not extend to several variables.

The orem 3. Suppose $\rho$ is a positive continuous function on $T^{N}$ and $f$ a zero-free function in $H^{\infty}\left(U^{N}\right)$ with $|f|^{*}(w)=\rho(w)$ for all $w \in T^{N}$. Then $\log \rho$ is in $R P\left(T^{N}\right)$.

Definition. If $f$ is a function on $U^{N}$ and $w \in T^{N}$, the "slice function" $f_{w}$ is defined on the unit disc by

$$
f_{w}(\lambda)=f(\lambda w) \quad(\lambda \in U) .
$$

Proof of Theorem 3. Let $f$ be any function in $H^{\infty}\left(U^{N}\right)$ with $\left|f^{*}\right|$ identically equal to $\rho$ on $T^{N}$. For each $w \in T^{N}$, the slice function $f_{w}$ is in $H^{\infty}(U)$ and $\left|f_{w}\right|$ has radial limits satisfying

$$
\left|f_{w}\right|^{*}(\lambda)=\rho(\lambda w)>0 \text { for all } \lambda \in T .
$$

Since the radial limit of a nonconstant singular inner function on $U$ must vanish at some point of $T$, it follows that the inner factor of $f_{w}$ is a Blaschke product [1, Chapter 5]. Hence, for each $w \in T^{N}$, the least harmonic majorant of $\log \left|f_{w}\right|$ is the Poisson integral $P\left[\log \left|f_{w}\right|^{*}\right]$. This implies, by [3, Theorem 3.3.6], that $P[\log \rho]$ is the least $N$-harmonic majorant of $\log |f|$ in $U^{N}$.

Now if $f$ is never zero in $U^{N}, \log |f|$ is its own least $N$-harmonic majorant. Hence $\log |f|=P[\log \rho]$ and it follows [3, p. 73] that $\log \rho \in R P\left(T^{N}\right)$.

The final theorem illustrates more dramatically the difference between the situations in one and several variables. In particular, it implies that if $A$ is the circle $\{(\zeta, \zeta):|\zeta|=1\}$ in $T^{2}$, there exists a positive continuous function $\rho$ on $T^{2}$ to which there corresponds no zero-free $f \in H^{\infty}\left(U^{2}\right)$ with $|f|^{*}=\rho$ a.e. on $T^{2}$ and everywhere on $A$.

Theorem 4. Suppose $f$ is a function in $H^{\infty}\left(U^{2}\right)$ with

$$
|f|^{*}(\zeta, \zeta)=1 \text { for all } \zeta \in T \text {, }
$$

and such that $f(\lambda, \lambda)$ never vanishes for $\lambda \in U$. Then $\left\|f_{w}^{*}\right\|_{\infty} \geq 1$ for all $w \in T^{N}$ (where $\left\|f_{w}^{*}\right\|_{\infty}$ is the essential supremum of $\left|f_{w}\right|^{*}$ on $T$ ).

Proof. Let $F(\lambda)=f(\lambda, \lambda)$ for $\lambda \in U$. Then $F \in H^{\infty}(U), F$ has no zeros in $U$, and by (18), $|F|^{*}=1$ everywhere on $T$. In particular, $F$ is a singular inner function. But, the radial limit of a nonconstant singular inner function must vanish at some point of $T$. So $F$ must be constant and, in particular,

$$
|f(0,0)|=|F(0)|=1 \text {. }
$$

Now suppose $\left\|f_{w}^{*}\right\|_{\infty}<1$ for some $w \in T^{N}$. Since $f_{w} \in H^{\infty}(U)$, it follows that $\left|f_{w}(\lambda)\right|<1$ for all $\lambda \in U$. In particular, $|f(0,0)|=\left|f_{w}(0)\right|<1$, which contradicts (19). 


\section{REFERENCES}

1. K. Hoffman, Banach spaces of analytic functions, Prentice-Hall, Englewood Cliffs, N.J., 1962. MR 24 \#A2844.

2. W. Rudin, Real and complex analys is, McGraw-Hill, New York, 1966. MR $35 \# 1420$.

3. - Function theory in polydiscs, Benjamin, New York, 1969. MR 41 \#501.

4. A. Zygmund, Trigonometrical series, 2 nd. rev. ed., Cambridge Univ. Press, New York, 1959. MR $21 \# 6498$.

DEPARTMENT OF MATHEMATICS, CLAREMONT MEN'S COLLEGE, CLAREMONT, CALIFORNIA 91711 